\title{
Depósito subconjuntival de óleo de silicone após cirurgia vitreorretiniana
}

\author{
Subconjunctival deposit of siliconeoil after vitreoretinal surgery
}

\author{
Leonardo Provetti Cunha ${ }^{1}$ \\ Hélio Paulo Primiano Júnior ${ }^{2}$ \\ Augusto Nakashima ${ }^{3}$ \\ João Arthur Trein Júnior ${ }^{4}$ \\ Ramom Coral Ghanem ${ }^{5}$ \\ Ruth Miyuki Santo ${ }^{6}$ \\ Yoshitaka Nakashima ${ }^{7}$
}

Trabalho realizado no Departamento de Oftalmologia do Hospital das Clínicas da Faculdade de Medicina da Universidade de São Paulo - USP - São Paulo (SP) Brasil.

${ }^{1}$ Médico Preceptor do Hospital das Clínicas da Faculdade de Medicina da Universidade de São Paulo - USP - São Paulo (SP) - Brasil.

${ }^{2}$ Médico Assistente do Setor de Retina e Vítreo do Hospital das Clínicas da Faculdade de Medicina da USP São Paulo (SP) - Brasil.

3 Acadêmico da Faculdade de Medicina da USP - São Paulo (SP) - Brasil.

${ }^{4}$ Médico Estagiário do Setor de Retina e Vítreo do Hospital das Clínicas da Faculdade de Medicina da USP São Paulo (SP) - Brasil.

5 Médico Estagiário do Setor de Retina e Vítreo do Hospital das Clínicas da Faculdade de Medicina da USP São Paulo (SP) - Brasil.

${ }^{6}$ Doutora; Médica Assistente do Setor de Córnea e Doenças Externas e Patologia do Hospital das Clínicas da Faculdade de Medicina da USP - São Paulo (SP) Brasil.

${ }^{7}$ Doutor; Médico Assistente do Setor de Retina e Vítreo do Hospital das Clínicas da Faculdade de Medicina da USP - São Paulo (SP) - Brasil.

Endereço para correspondência: Leonardo Provetti Cunha. Rua Loefgren, 441/114 - São Paulo (SP) CEP 04040-000

E-mail: leonardo_provetti@yahoo.com.br.

Recebido para publicação em 01.08.2006 Última versão recebida em 27.02.2007 Aprovação em 02.04.2007

Nota Editorial: Depois de concluída a análise do artigo sob sigilo editorial e com a anuência do Dr. João Carlos Miranda Gonçalves sobre a divulgação de seu nome como revisor, agradecemos sua participação neste processo.

\section{RESUMO}

Objetivo: Comprovar a presença do óleo de silicone no espaço subconjuntival de pacientes submetidos previamente à cirurgia vitreorretiniana por meio de estudo histopatológico das amostras conjuntivais obtidas, nos quais o exame biomicroscópico não foi capaz de comprovar sua presença. Determinar qual a incidência da presença do óleo de silicone no espaço subconjuntival em uma série de casos e quais implicações clínicopatológicas. Métodos: Estudo prospectivo em 30 olhos de 30 pacientes. Foram incluídos no estudo os pacientes que haviam sido submetidos previamente à cirurgia vitreorretiniana com implante intra-ocular de óleo de silicone e que possuíssem indicação para retirada do óleo de silicone e que não apresentassem ao exame biomicroscópico sinais da presença do óleo de silicone no espaço subconjuntival. Após sua retirada, a amostra era encaminhada para análise histopatológica pelo método de hematoxilinaeosina. Resultados: Foi observada a presença de espaços vazios correspondentes às áreas de localização do óleo de silicone, removido durante processamento histológico, em 10 (33\%) amostras. Observou-se também a presença de sinais inflamatórios na substância própria caracterizada por congestão vascular, leucostase e infiltrado linfomononuclear em $27(90 \%)$ amostras. Conclusão: Portanto, em pacientes submetidos ao implante intra-ocular do óleo de silicone, devemos suspeitar que o óleo esteja presente no espaço subconjuntival, mesmo que o exame biomicroscópico pareça normal.

Descritores: Óleos de silicone/efeitos adversos; Retina/cirurgia; Corpo vítreo/cirurgia Biopsia; Cuidados pós-operatórios

\section{INTRODUÇÃOO}

A cirurgia de vitrectomia posterior associada ao implante intra-ocular de óleo de silicone permitiu maior êxito na aplicação anatômica da retina descolada quando outros procedimentos falharam. Entretanto, inúmeras complicações associadas ao uso intra-ocular de silicone são relatadas na literatura $^{(1-6)}$, tais como catarata, ceratopatia em faixa, hipertensão ocular, emulsificação do óleo de silicone, uveíte crônica, dor ocular, proliferação fibrovascular, membranas pupilares, endoftalmite e depósito subconjuntival de óleo de silicone ${ }^{(1,7)}$. O extravasamento do óleo de silicone para o espaço subconjuntival é, portanto, uma das complicações possíveis ${ }^{(1-3,5)}$.

O diagnóstico dos depósitos subconjuntivais do óleo de silicone poderá ser feito por meio do exame biomicroscópico. Pode ser observado como nódulos subconjuntivais translúcidos, de tamanho variável, únicos ou múltiplos. Entretanto, acreditamos que alguns pacientes possam apresentar estes depósitos na conjuntiva e os mesmos não serem visíveis à biomicroscopia. 
Portanto, no estudo atual nos propomos a comprovar a presença do óleo de silicone no espaço subconjuntival de pacientes submetidos previamente à cirurgia vitreorretiniana por meio de estudo histopatológico das amostras conjuntivais obtidas destes pacientes, nos quais o exame biomicroscópico não fosse capaz de comprovar sua presença. Nos propomos também, a determinar qual a incidência da presença do óleo de silicone no espaço subconjuntival em uma série de casos e quais implicações clínico-patológicas destes achados.

\section{MÉTODOS}

Foi realizado um estudo prospectivo em 30 olhos de 30 pacientes. Este estudo foi aprovado pela Comissão de Ética para Análise de Projetos de Pesquisa (CAPPesq) da Diretoria Clínica do Hospital das Clínicas da Faculdade de Medicina da Universidade de São Paulo. Foram incluídos no estudo os pacientes que haviam sido submetidos previamente à cirurgia vitreorretiniana com implante intra-ocular de óleo de silicone e que possuíssem indicação para retirada do óleo de silicone. Foram incluídos somente os pacientes que não apresentassem ao exame biomicroscópico prévio, sinais da presença do óleo de silicone no espaço subconjuntival. As amostras conjuntivais eram obtidas no intra-operatório, durante a abertura da conjuntiva, antes da realização das esclerotomias, geralmente na região temporal inferior, próxima ao local da esclerotomia prévia, utilizada para fixação da infusão. As amostras conjuntivais deveriam medir aproximadamente 3 milímetros de diâmetro. Após sua retirada, a amostra era conservada em solução de formol e encaminhada para análise histopatológica.

\section{RESULTADOS}

Foram analisadas as amostras conjuntivais pelo método de hematoxilina-eosina de 30 olhos de 30 pacientes consecuti- vos. Foi observada a presença de espaços vazios correspondentes às áreas de localização do óleo de silicone, removido durante processamento histológico em 10 amostras (33\%) (Figuras 1 e 2). Dezoito amostras (60\%) foram consideradas negativas para presença do óleo de silicone e em 2 amostras (7\%) o resultado foi considerado indeterminado. O gráfico 1 mostra a distribuição dos resultados obtidos. Observou-se também a presença de sinais inflamatórios na substância própria caracterizada por congestão vascular, leucostase e infiltrado linfomononuclear em 27 amostras (90\%) (Figura 2 e Gráfico 2). Não foi observada em nenhuma amostra a presença de reação inflamatória do tipo granulomatosa.

\section{DISCUSSÃO}

O uso de óleo de silicone é aceito como procedimento seguro e efetivo no tratamento dos descolamentos de retina complicados, permitindo que a retina fique aplicada enquanto outros procedimentos falharam ${ }^{(7)}$. Federman et al. ${ }^{(1)}$ descreveram as complicações associadas ao uso de óleo de silicone em 150 olhos após cirurgia vitreorretiniana. 2,7\% destes olhos apresentaram ao exame biomicroscópico depósito subconjuntival de óleo de silicone associado à dor ocular. Entretanto, no presente estudo, a presença do óleo de silicone na conjuntiva foi demonstrada em 33\% dos pacientes. Estes valores são bem superiores aos encontrados por Federman et al. ${ }^{(1)}$. Acreditamos que este maior índice de positividade deve-se ao fato de que a biópsia conjuntival sugere ser mais sensível em demonstrar a presença do óleo de silicone na conjuntiva quando comparada ao exame biomicroscópico apenas.

O óleo de silicone pode extravasar pela esclerotomia durante a infusão e/ou no momento da sutura da esclerotomia. A irrigação copiosa com soro fisiológico após o procedimento pode não ser capaz de remover completamente o óleo de silicone. A sua remoção pode ser difícil porque o óleo torna-se

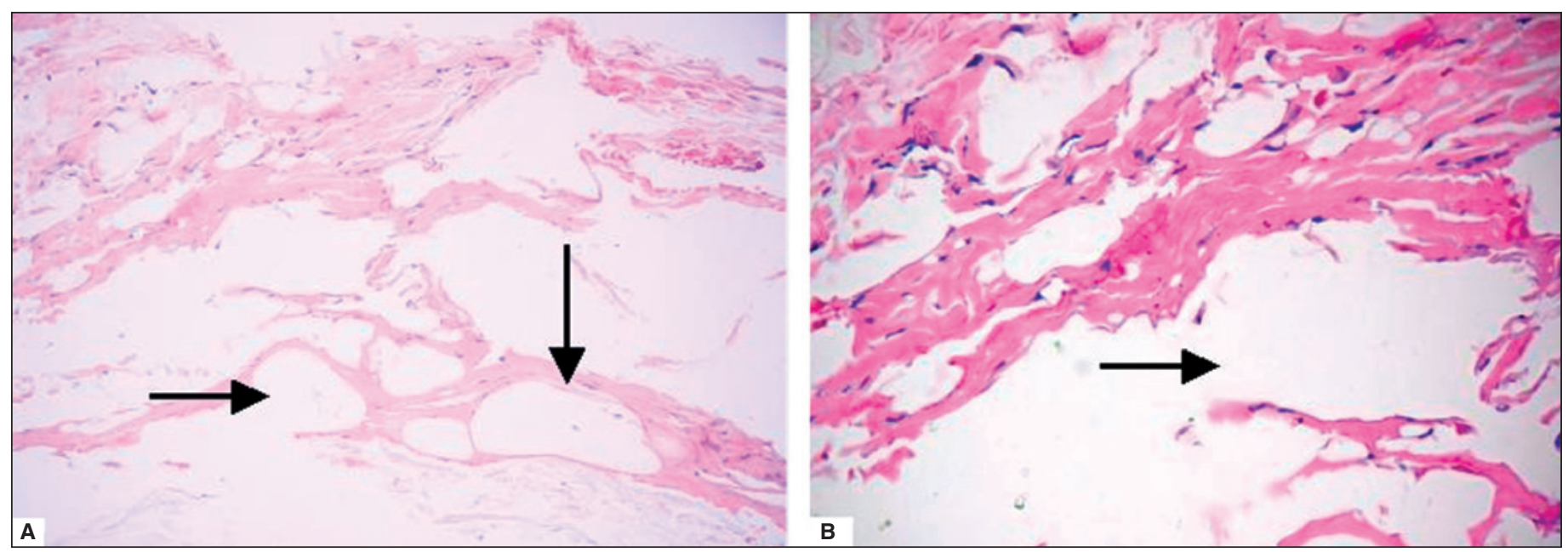

Figura 1 - Biópsia conjuntival evidenciando espaços vazios correspondentes às áreas de localização do óleo de silicone (setas). (Hematoxilinaeosina, A X200 e B X400) 


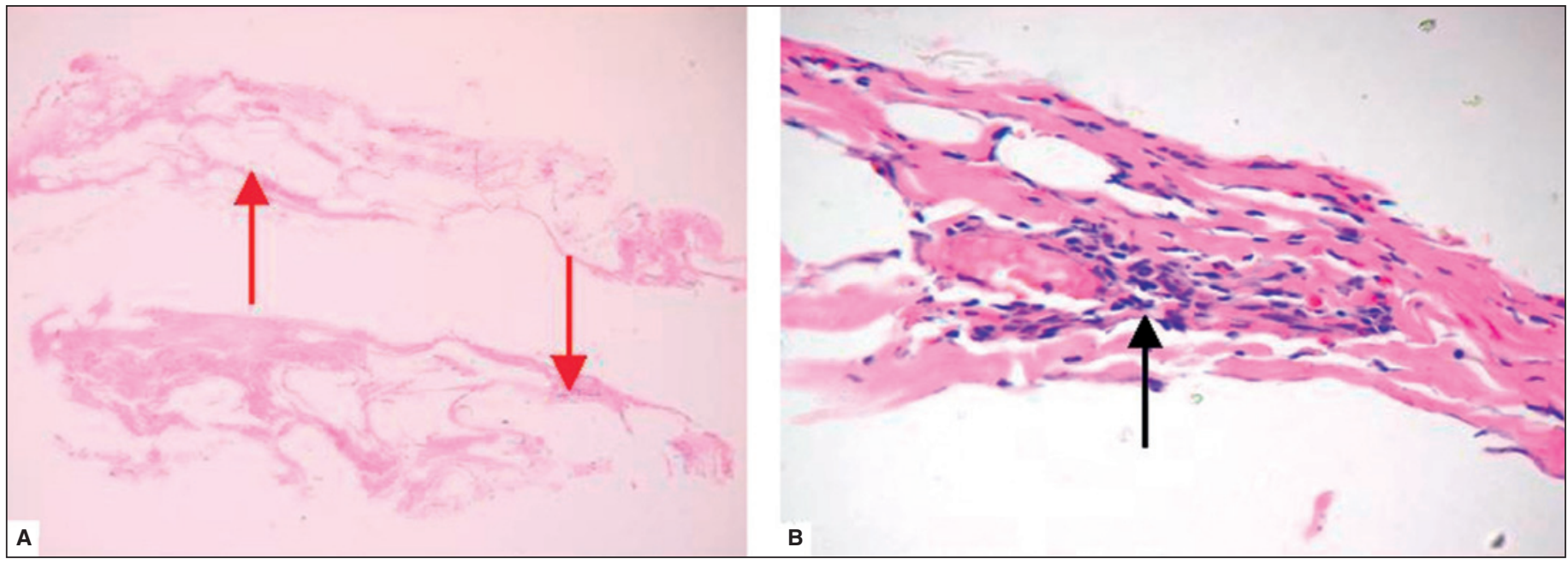

Figura 2 - Biópsia conjuntival: espaços vazios correspondentes às áreas de localização do óleo de silicone, removido durante processamento histológico (setas vermelhas), infiltrado inflamatório na substância própria com congestão vascular, leucostase e infiltrado linfomononuclear (seta preta). (Hematoxilina-eosina, A X40 e B X400).

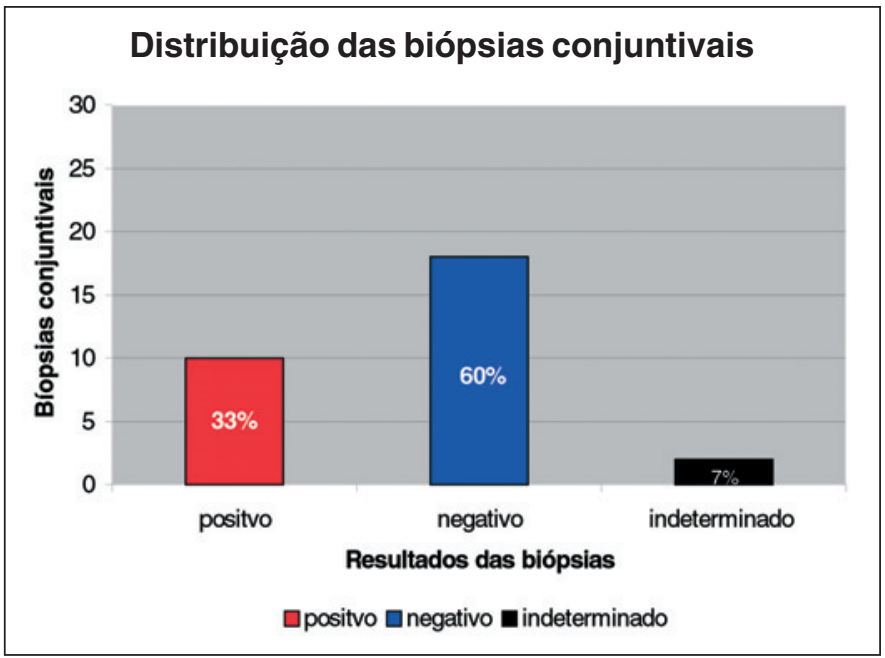

Gráfico 1 - Distribuição das biópsias conjuntivais analisadas pelo método de hematoxilina-eosina quanto à presença do óleo de silicone

multiloculado, favorecendo a resposta lipogranulomatosa nos espaços do tecido conectivo episcleral.

No presente estudo, a dor ocular foi o sintoma mais freqüente, presente em 21 (70\%) pacientes. A ocorrência da dor pode ser justificada pela própria resposta inflamatória gerada no pós-operatório, podendo ser exacerbada pela presença do óleo de silicone no espaço subconjuntival. Outros estudos serão necessários a fim de melhor avaliar estes achados.

Nakashima et al. ${ }^{\left({ }^{8}\right)}$, relataram o caso de uma paciente que apresentou cisto subconjuntival de óleo de silicone após vitrectomia posterior via pars plana, associada à dor ocular e deformidade estética, onde o tratamento definitivo foi sua remoção cirúrgica. Outro relato semelhante ${ }^{(9)}$, também demonstrou a presença de cisto subconjuntival de óleo de silicone com análise histopatológica, evidenciando infiltrado fibro-

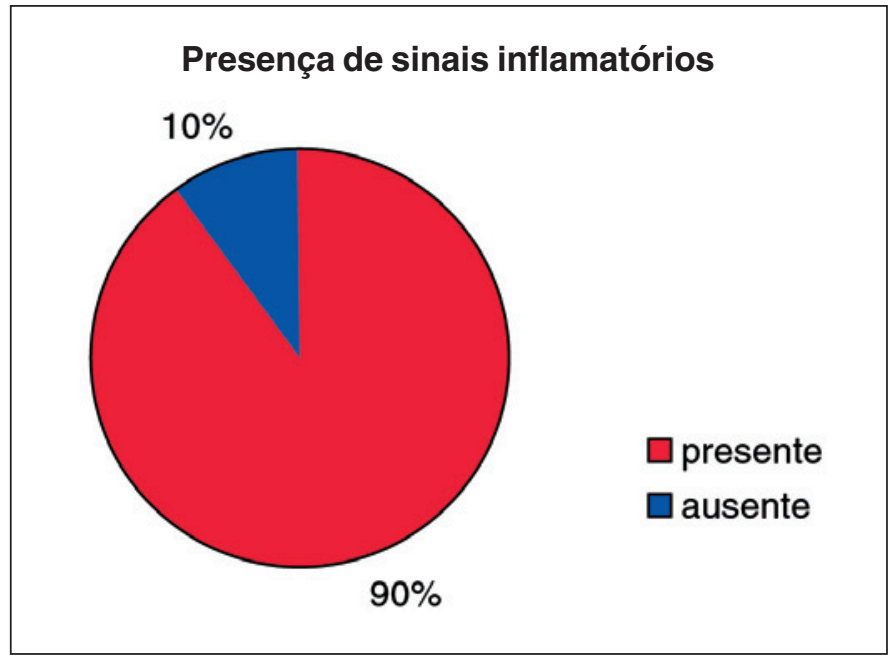

Gráfico 2 - Distribuição das biópsias conjuntivais analisadas pelo método de hematoxilina-eosina quanto à presença de sinais inflamatórios

vascular inflamatório crônico do tipo granulomatoso com presença de células gigantes, sendo esta a única publicação onde foi realizado estudo histopatológico de cisto subconjuntival por óleo de silicone. No presente estudo, em nenhuma das amostras foi observada a presença de reação inflamatória do tipo granulomatosa com formação de células gigantes.

Estes autores ${ }^{(10)}$, relataram que as moléculas de óleo de silicone de baixo peso molecular são capazes de difundir através dos tecidos, levando a uma reação tóxica e/ou inflamatória. No presente estudo, infiltrados inflamatórios estiveram presentes em 27 (90\%) amostras (Gráfico 2), mesmo para as amostras com resultados negativos para a presença do óleo, podendo tratar-se de um viés de amostragem, sendo possível que o óleo de silicone estivesse presente em outras partes da conjuntiva não biopsiada. Ressaltamos também que a própria 
agressão provocada pelo procedimento cirúrgico pode por si só justificar a presença de reação inflamatória crônica.

\section{CONCLUSÃO}

No presente estudo, a comprovação histopatológica da presença de óleo de silicone nas amostras conjuntivais foi de $33 \%$. Portanto, em pacientes submetidos ao implante intra-ocular do óleo de silicone devemos suspeitar que o óleo esteja presente na conjuntiva ou no espaço subconjuntival, mesmo que o exame biomicroscópico pareça normal. De acordo com o nosso conhecimento, trata-se do primeiro estudo que teve como objetivo determinar qual a incidência da presença do óleo de silicone no espaço subconjuntival em uma série de casos e quais as implicações clínico-patológicas destes achados. O oftalmologista deverá estar atento a esta possível complicação após o uso intra-ocular de óleo de silicone em cirurgia vitreorretiniana.

\section{ABSTRACT}

Purpose: To assess the histopathological findings of conjunctival specimens from patients submitted to vitreoretinal surgery with intraocular silicone oil injection. Methods: Prospective analyses of 30 eyes of 30 patients were evaluated. Before the patients underwent removal of the intraocular silicone oil, conjunctival excision was performed and submitted to histopathologic examination. Results: The presence of empty spaces corresponding to silicone oil location was positive in $10(33 \%)$ specimens. The presence of inflammatory cells, vascular congestion, leukostases, lymphocyte and monocyte infiltrates were positive in $27(90 \%)$ specimens. The presence of silicone oil was positive in 10 (33\%) specimens. Therefore, in those patients who undergo vitreoretinal surgery, silicone oil may be present in the conjunctiva or subconjunctival space, even if biomicroscopic examination seems to be normal. According to our knowledge, this is the first study with the purpose to assess the histopathological findings of conjunctival specimens from patients submitted to vitreoretinal surgery with intraocular silicone oil injection. Conclusion: Ophthalmologists should be aware of this possible complication after intraocular use of silicone oil after vitreoretinal surgery.

Keywords: Silicone oils/adverse effects; Retina/surgery; Vitreous body/surgery; Biopsy; Postoperative care

\section{REFERÊNCIAS}

1. Federman JL, Schubert HD. Complications associated with the use of silicone oil in 150 eyes after retina-vitreous surgery. Ophthalmology. 1988;95(7):870-6.

2. Hyung SM, Min JP. Subconjunctival silicone oil drainage through the Molteno implant. Korean J Ophthalmol. 1998;12(1):73-5.

3. Nazemi PP, Chong LP, Varma R, Burnstine MA. Migration of intraocular silicone oil into the subconjunctival space and orbit through an Ahmed glaucoma valve. Am J Ophthalmol. 2001;132(6):929-31.

4. Honda Y, Ueno S, Miura M, Yamaguchi H. Silicone oil particles trapped in the subretinal space: complications after substitution of the vitreous. Ophthalmologica. 1986;192(1):1-5.

5. Biswas J, Bhende PS, Gopal L, Parikh S, Badrinath SS. Subconjunctival cysts following silicone oil injection: a clinicopathological study of five cases. Indian J Ophthalmol. 1999;47(3):177-80.

6. Torres RJA, Luchini A, Torres RJA, Abib FC, Torres RAA. Prótese de íris, na aniridia traumática, como tentativa de controlar glaucoma refratário provocado pela presença de óleo de silicone na câmara anterior: relato de caso. Arq Bras Oftalmol. 2005;68(3):389-92.

7. Cockerham WD, Schepens CL, Freeman HM. Silicone injection in retinal detachment. Arch Ophthalmol. 1970;83(6):704-12.

8. Nakashima Y, Souza EC, Oliveira BFT, Shida TM. Cisto de óleo de silicone subconjuntival após vitrectomia. In: $3^{\circ}$ Congresso de Oftalmologia USP; $2^{\circ}$ Congresso de Auxiliar de Oftalmologia. São Paulo; 2000.

9. Hutchinson AK, Capone A, Grossniklaus HE. Subconjunctival silicone oil after vitreoretinal surgery. Am J Ophthalmol. 1993;115(1):109-10.

10. Gabel VP, Kampik A, Burkhardt J. Analysis of intraocularly applied silicone oils of various origins. Graefes Arch Clin Exp Ophthalmol. 1987;225(3):160-2. 